Explosive Pulsed Power Experimental Capability at LLNL

A. D. White, D. P. Milhous, D. A. Goerz, R. A. Anderson, T. J. Ferriera, R. D. Speer, R. M. Kuklo, A. J. Young, D. A. Lahowe, G. R. Mease, M. A. Suda, A. D. Wiltse, D. B. Reisman, E. P. Daykin

November 13, 2012

IEEE 14th International Conference on Megagauss Magnetic Field Generation and Related Topics Maui, HI, United States October 14, 2012 through October 19, 2012 
This document was prepared as an account of work sponsored by an agency of the United States government. Neither the United States government nor Lawrence Livermore National Security, LLC, nor any of their employees makes any warranty, expressed or implied, or assumes any legal liability or responsibility for the accuracy, completeness, or usefulness of any information, apparatus, product, or process disclosed, or represents that its use would not infringe privately owned rights. Reference herein to any specific commercial product, process, or service by trade name, trademark, manufacturer, or otherwise does not necessarily constitute or imply its endorsement, recommendation, or favoring by the United States government or Lawrence Livermore National Security, LLC. The views and opinions of authors expressed herein do not necessarily state or reflect those of the United States government or Lawrence Livermore National Security, LLC, and shall not be used for advertising or product endorsement purposes. 


\title{
Explosive Pulsed Power Experimental Capability at LLNL
}

\author{
A. D. White, D. P. Milhous, D. A. Goerz, R. A. Anderson, T. J. Ferriera, R. D. Speer, R. M. Kuklo, A. J. \\ Young, D. A. Lahowe, G. R. Mease, M. A. Suda, A. D. Wiltse, and D. B. Reisman \\ Lawrence Livermore National Laboratory \\ Livermore, USA \\ $<$ E-mail>white210@1lnl.gov \\ E. P. Daykin \\ National Security Technologies \\ Las Vegas, USA
}

\begin{abstract}
LLNL has developed a family of advanced magnetic flux compression generators (FCGs) used to perform high energy density physics experiments and material science studies. In recent years we have performed these experiments at explosive test sites in New Mexico and Nevada. In 2011, we re-established an explosive pulsed power test facility closer to Livermore. LLNL's Site 300 is a U.S. DOE-NNSA experimental test site situated on 7000 acres in rural foothills approximately 15 miles southeast of Livermore. It was established in 1955 as a non-nuclear explosives test facility to support LLNL's national security mission. On this site there are numerous facilities for fabricating, storing, assembling, and testing explosive devices. Site $\mathbf{3 0 0}$ is also home to some of DOE's premier facilities for hydrodynamic testing, with sophisticated diagnostics such as high-speed imaging, flash $X$-ray radiography, and other advanced diagnostics for performing unique experiments such as shock physics experiments, which examine how materials behave under high pressure and temperature. We have converted and upgraded one particular firing bunker at Site 300 (known as Bunker 851) to provide the necessary infrastructure to support high explosive pulsed power (HEPP) experiments. In doing so, we were able to incorporate our established practices for handling grounding, shielding, and isolation of auxiliary systems and diagnostics, in order to effectively manage the large voltages produced by FCGs, and minimize unwanted coupling to diagnostic data. This paper will discuss some of the key attributes of the Bunker 851 facility, including the specialized firesets and isolated initiation systems for multistage explosive systems, a detonator-switched seed bank that operates while isolated from earth and building ground, a fiber-optic based timing, triggering and control system, an EMI Faraday cage that completely encloses diagnostic sensors, cabling and high-resolution digitizers, optical fiber-based velocimetry and current sensor systems, and a flash $X$-ray radiography system. The photos and experimental results from recent FCG experiments will also be shown and discussed.
\end{abstract}

Keywords—flux compression generator; explosive pulsed power

\section{INTRODUCTION}

A facility capable of firing HEPP experiments has several requirements that exceed the requirements of a conventional HE experimental facility. There are constraints on facility system design, due to the need to manage voltages and radiated electromagnetic energy produced during flux compression generator operation, and there are capabilities necessary to fire and diagnose the generator itself. A successful HEPP facility will provide the necessary capabilities, while incorporating design principles that manage the constraints.

Our successful approach to mitigating the unique constraints of HEPP facility design is two-fold: 1) understand the voltages that will be developed between components within the experiment, and incorporate high-voltage isolation into the system design for affected systems, and 2) understand how radiated EM energy from the experiment will couple to systems and diagnostics within the experiment; when necessary use a Faraday cage or isolation via optical fiber to minimize and manage unwanted coupling. These two grounding, shielding and isolation design principles were incorporated into the design of the Bunker 851 HEPP complex

The capabilities necessary to fire our two-stage flux compression generators can be summarized with five requirements: 1) a timing and firing system (that perhaps closely resembles a conventional HE timing and firing 
system), 2) a capacitor seed bank capable of delivering roughly 60-80 kA into the initial $10 \mu \mathrm{H}$ inductance of our generator pair, 3) multi-stage initiation systems to detonate exploding bridge-wires that initiate HE in the generators (and to fire a detonator-switched seed bank switch), 4) specialized diagnostics such as Faraday rotation current diagnostics, photonic doppler velocimetry (PDV) sensors, and X-ray imaging systems, and the data acquisition systems necessary to record these signals and, 5) A Faraday cage to provide EMI shielding for conventional pulsed power diagnostic signals, and the associated data acquisition hardware. As mentioned, the design of each of these systems must incorporate the grounding and shielding and isolation design principles described in the preceding paragraph.

\section{SYSTEM DESCRIPTIONS}

Systems meeting the above requirements have been designed, fabricated, and operated at Bunker 851 , and will be described in the following section in detail. A CAD model image of the Bunker 851 complex is shown in Fig. 1, and illustrates the relative location of the pertinent systems.

\section{A. Timing, Firing and Control Systems}

The timing, firing and control systems closely resemble those used for conventional hydrodynamic testing. To meet sub-system isolation requirements, trigger and fiducial signals are distributed over optical fiber. Control system signals are distributed over optical fiber or pneumatic airline. Outgoing trigger signals are sampled at generation and recorded on high-speed digitizers to verify trigger and fiducial timing on every dry-run and shot.

\section{B. An Explosively Switched Seed Bank}

A capacitor bank was designed and fabricated to produce the initial generator current of $60 \mathrm{kA}, 80 \mathrm{kA}$ or $140 \mathrm{kA}$ in our FPG [1], Mini-G [2], and AHG [3] or FFT [4] systems, respectively, and is shown in Fig. 2. The capacitor bank consists of either a single $300 \mu \mathrm{F}$ capacitor for FPG or Mini-G experiments or four $300 \mu \mathrm{F}$ capacitors arranged in parallel for either FFT or AHG experiments. At shot time, relays isolate the seed bank from facility ground. During this time, the seed bank is powered from a UPS.

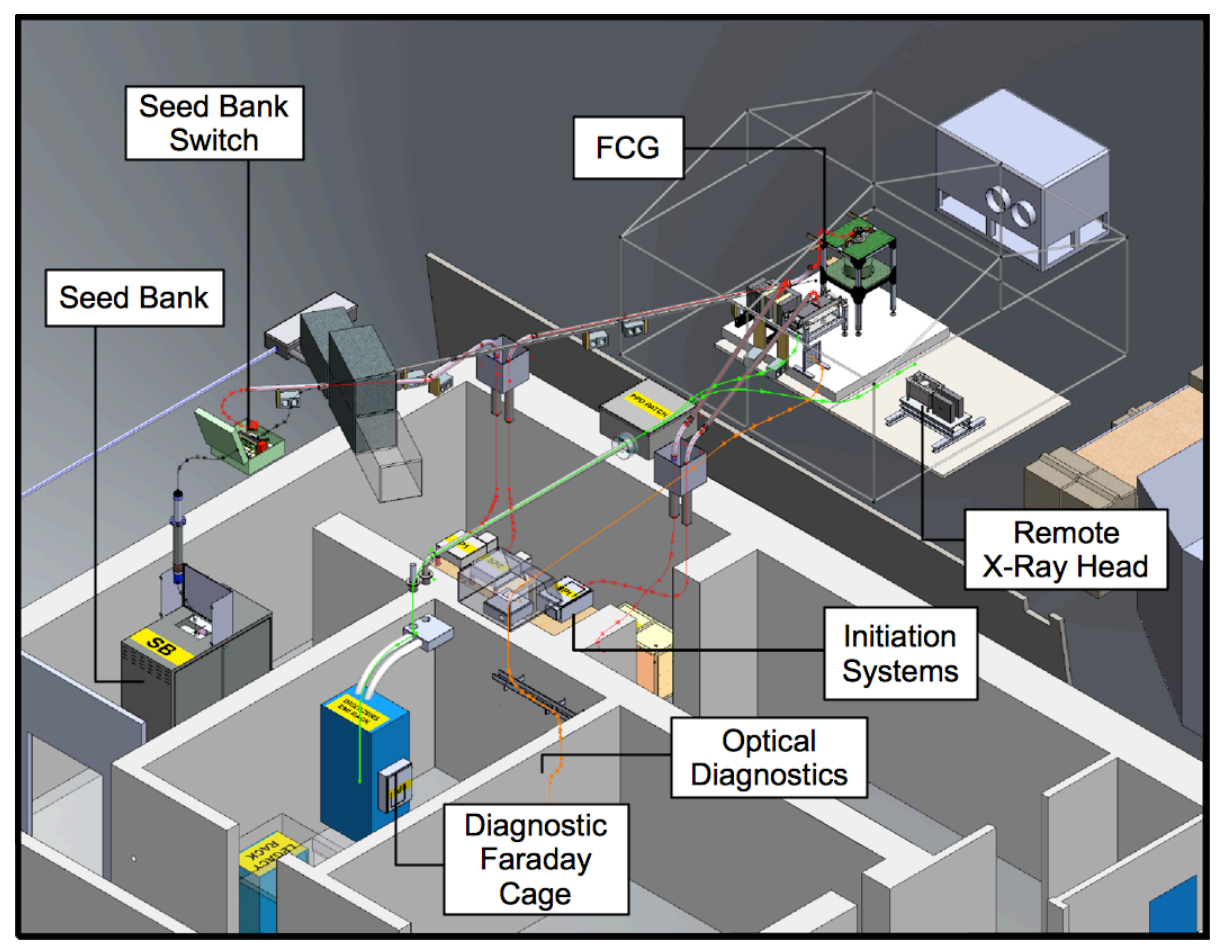

Figure 1. An image from a CAD model of the Bunker 851 complex, illustrating the relative layout of HEPP systems discussed in this paper.

Four YK-198 cables connect the seed bank output to an LLNL-designed explosively-driven dielectric puncture switch. The switching action is accomplished by detonating three RP-87 detonators arranged in series within the switch using a dedicated fireset. During early experiments, the detonator cable was isolated from the switch, but 
became connected intermittently during switch operation, causing high-frequency transient currents to flow to the otherwise isolated fireset. Subsequently, we connected the low-side of the detonator cable to the high-side of the switch through an intentional low impedance connection. During seed bank operation, this connection causes the fireset to float to the potential of the switch relative to ground (which is innocuous provided the high-voltage isolation of the fireset is sufficient), but significantly reduces the high frequency transients that affect other data (see IS\#1 in Fig. 6).

During FCG operation, the seed bank, detonator switch, and seed bank cables are elevated to the potential of the top of the helical coil relative to ground. The magnitude of this voltage is approximately $20 \mathrm{kV}$ for a Mini-G, and 40 $\mathrm{kV}$ for an FFT [5]. The system was designed to withstand $50 \mathrm{kV} \mathrm{DC}$ isolation from facility ground with this phenomenon in mind. We verify this $50 \mathrm{kV}$ DC isolation with a high-potter before each experiment.

The seed bank current and voltage are recorded, as is the switch output current. The electric potential developed between the seed bank and facility ground is also recorded. Due to isolation requirements, all diagnostic signals originating at the seed bank are converted to optical signals and routed over optical fiber to recording digitizers which are located elsewhere in the bunker.

\section{Four Isolated Initiation Systems}

We use four initiation systems (IS) to initiate HE: IS \#1 is a $1 \mu \mathrm{F}$ single-point fireset that is charged to $3.5 \mathrm{kV}$ to fire the three RP-87 detonators in the seed bank switch (see Fig. 2), IS \#2 is an identical $1 \mu \mathrm{F}$ single-point fireset that is charged to $3.5 \mathrm{kV}$ to initiate a single RP-1 reduced load detonator in the helical generator, IS \#3 is a $120 \mu \mathrm{F}$ ninepoint fireset that is charged to $2 \mathrm{kV}$ to initiate eight RP-1 reduced load detonators on the FPG, and IS \#4 is a $14 \mu \mathrm{F}$ 54-point fireset that is charged to $10 \mathrm{kV}$ to initiate $51 \mathrm{RP}-1$ reduced load detonators (FFT) or $51 \mathrm{RP}-2$ detonators (Mini-G) (see Fig. 3).

IS\#1, IS\#2 and IS\#3 are all switched using spark gaps; IS\#4 is switched using a detonator switch that is fired with a CDU located within IS\#4.

The configuration of IS \#4 differs between FFT and Mini-G experiments: on a Mini-G, the detonators are arranged in 3-in-series arrangements, so that only 17 of the fireset outputs are needed. On an FFT experiment, one output each is used for the 51 detonators.
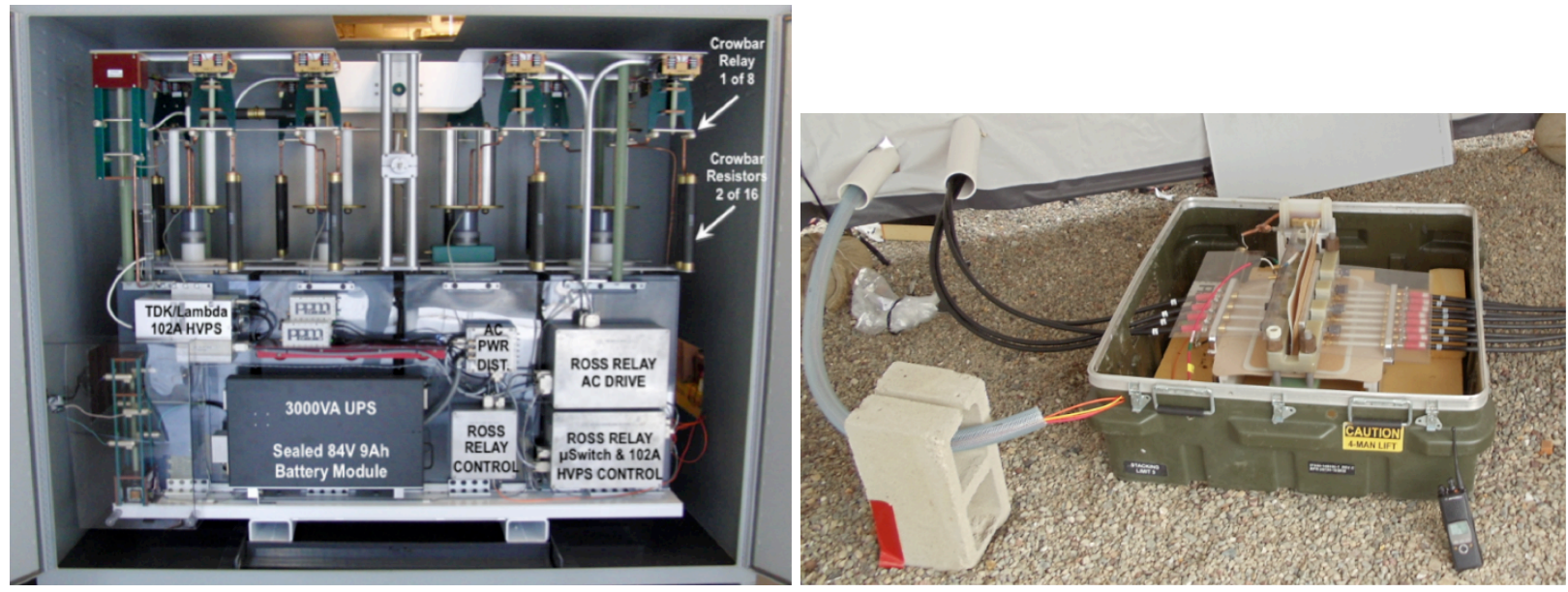

Figure 2. (left) Photograph illustrating the seed bank hardware. Relays are used to isolate the seed bank from facility ground at shot time, at which point a UPS is used to provide system power. Crowbar relays are used to crowbar the capacitors for safety purposes after a shot. (right) Photograph of the LLNL-designed seed bank explosive dielectric puncture switch. A red "C" cable and several optical fibers connect detonators and diagnostics in the switch enclosure to the fireset and associated hardware in the bunker. Eight YK-198 cables connect the input of the switch

on the right to the seed bank; four YK-198 cables connect the output of the switch on the left to the helical generator input. An RC snubber circuit is installed across the output of the switch, in the aft of the switch enclosure. The RC snubber slows the risetime of the voltage waveform across the switch output, which reduces the amplitude of transient voltages that appear at the helical generator input due to impedance mismatch. 

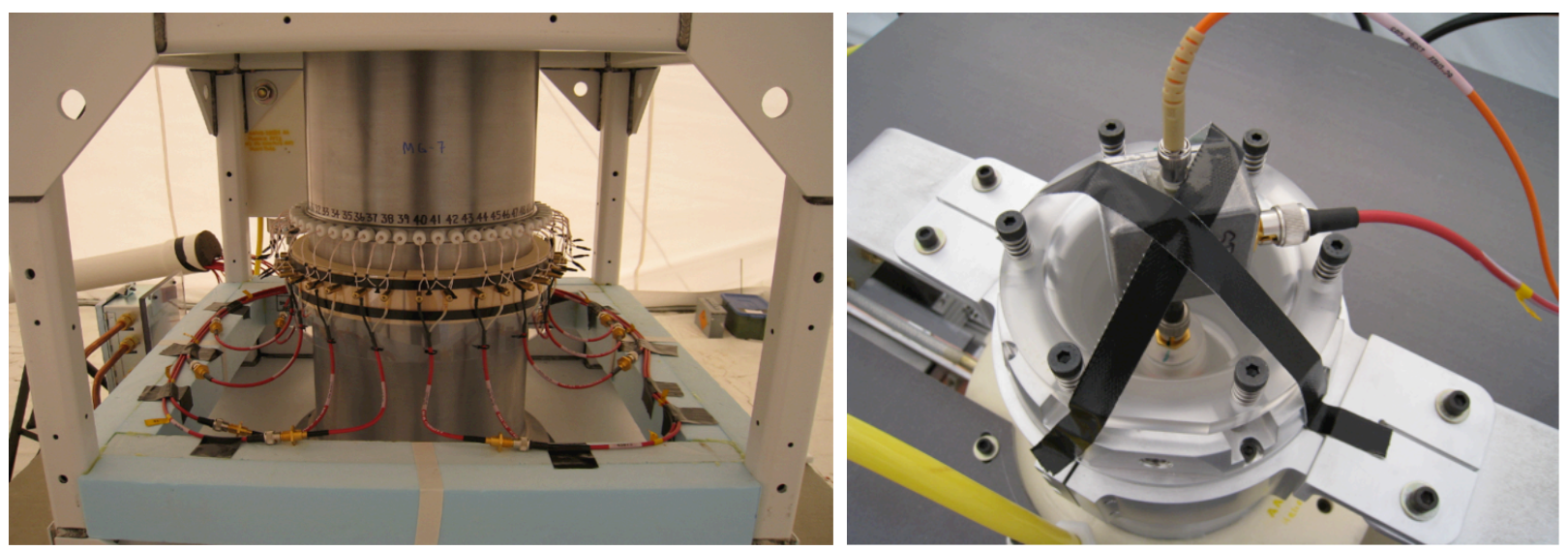

Figure 3. (left) A photograph of 17 " $\mathrm{C}$ " cables connected to 51 RP-1 reduced load detonators (in 3-in-series arrangement) used to initiate the HE around the waist of a Mini-G. (right) The single-point initiation at the top of the helical generator. An optically-coupled voltage probe is connected directly between the fireset "C" cable and the detonator.

One additional output of both IS \#3 and IS \#4 is used for diagnostic purposes only: a cable identical to the cables used on the other outputs is connected to detonator(s) identical to the detonator(s) used on other outputs, but instead of initiating HE, it is connected to an optically-coupled voltage probe and a bare optical fiber. The voltage probe is used to detect the time of bridge-wire burst, while the bare optical fiber is used to determine the time of HE breakout from the detonator. The fireset current is also recorded on both IS \#3 and IS \#4, and similar to the seed bank diagnostics, the signals are coupled to recording digitizers elsewhere in the bunker over optical fiber. The fireset current waveforms are used to verify burst timing and nominal fireset performance.

Leakage flux from the FCG can couple to loops formed between the cable shields of fireset cables and facility ground, elevating the firesets to several $\mathrm{kV}$ in potential. Thus, we apply the same $50 \mathrm{kV}$ DC isolation rule to the firesets, and verify this with hi-potting prior to each experiment.

\section{Specialized Diagnostic Capability}

Optical-fiber based Faraday Rotation diagnostics are our primary means of measuring FCG current [6, 7]. By looping our sensing fibers around the current carrying conductors in our generators an integer number of times, Ampere's law can be exploited to relate the observed optical rotation to generator current without any in situ calibration or geometric corrections. These sensors are exceptionally immune to EMI, and since the sensors are entirely dielectric in the vicinity of the generator, they pose no grounding and shielding concerns.

We place the Faraday rotation polarization analysis hardware in the bunker in order to protect it from the HE blast. To maintain an isotropic medium between the sensor fiber and the polarization analysis hardware, we use the same twisted optical fiber that we use in the sensor itself. We have found it convenient to order this optical fiber from an outside vendor [8], and replace the fiber between each shot.

We use both conventional and multiplexed photonic Doppler velocimetry (PDV) systems $[9,10]$ to measure surface velocities on our experiments. Similar to the Faraday rotation diagnostics, these are entirely dielectric between the bunker and FCG, so grounding and shielding concerns are negligible.

To provide X-ray imaging of FCG operation, we use $300 \mathrm{kV}$ and $450 \mathrm{kV}$ portable Scandiflash Marx pulsers to drive a remote X-ray head. The Marx pulsers are located in the bunker, while the X-ray head and the recording media are located on the shot table in close vicinity to the generator. Both the X-ray head and the recording media are blastprotected (see Fig. 4). The Marx pulser current and timing are diagnosed with a current viewing resistor installed at the Marx output. A PIN-diode installed in the vicinity of the X-ray head provides X-ray dosage and timing data. 


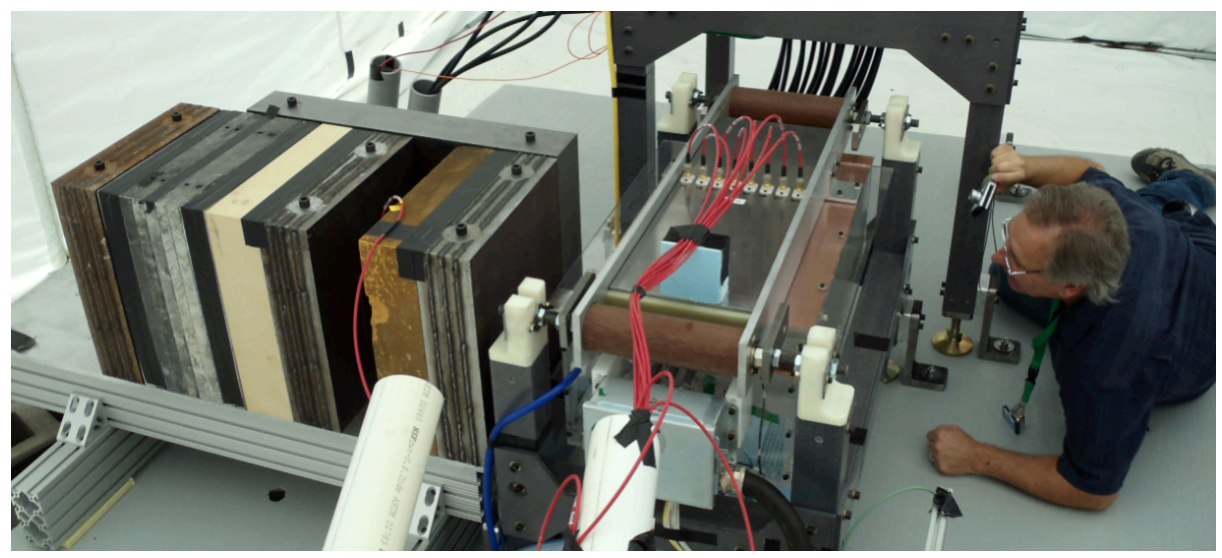

Figure 4. A FPG on the firing table. The X-ray imaging plates are within the layered blast protection to the left of the generator. A single red "C" cable, which exits the large PVC pipe in the foreground, fires a detonator that closes a blast shutter during the experiment to protect the

X-ray recording media. The eight " $\mathrm{C}$ " cables attached to the top of the generator are connected to detonators that initiate the FPG HE.

\section{E. A Faraday Cage and Isolated Data Acquisition System for High Dynamic Range Conventional Pulsed Power Diagnostic Measurements}

In order to minimize unwanted coupling to our B-dot measurements, we have found it necessary to route the B-dot signals entirely within a Faraday cage between the FCG and the recording digitizers located within the bunker. The Faraday cage at Bunker 851 was constructed out of solid steel pipe wherever possible. Flexible Glenair ${ }^{\mathrm{TM}}$ conduit is used wherever steel conduit is mechanically or operationally impractical. We perform current injection measurements of our Faraday cage, and by measuring the current injected on the exterior of the Faraday cage, and the current coupled to the shields of the coaxial cables within the Faraday cage, can derive a metric for the shielding effectiveness of the Faraday cage. In the frequencies of seed bank operation and FCG operation $(1 \mathrm{kHz}-1 \mathrm{MHz})$, we observe a shielding effectiveness of greater than $75 \mathrm{~dB}$. A thorough description of our Faraday cage, diagnostics, and measurement techniques has been published [6].

To further diagnose unwanted EMI coupling during the experiment, we install a null B-dot that consists of a cable and data acquisition hardware identical to the functioning B-dots, but with a short installed in lieu of a B-dot loop. During FCG experiments, we record this signal, and compare with the voltages produced by the functioning B-dots. The voltage measured on the null signal is typically two to four orders of magnitude lower than the voltages measured by the functioning B-dot probes (see Fig. 7).

In order to increase the dynamic range of our B-dot measurements, the B-dot signal is split between three digitizer channels of varying sensitivity. Care must be taken to avoid introducing an impedance mismatch when a sensitive channel clips, and the digitizer begins to clamp, as this will affect other channels that are still recording on scale. We have found that ZTEC Instruments [11] ZT4441 digitizers are particularly well suited to our application, since 1) the ZT4441 will not create an impedance mismatch due to overdrive regardless of full-scale voltage setting until $6 \mathrm{~V}$ is applied to the input, and 2) the trade-off between time resolution and ADC resolution is suitable for our application with $400 \mathrm{MS} / \mathrm{s}$ sample rates and 14-bit ADC resolution. Typical dynamic ranges of for our B-dot measurement are around $88.2 \mathrm{~dB}$ using the hardware and practices described above.

In addition to the aforementioned diagnostics, we frequently use current monitors to measure currents coupled into our bunker Faraday cages and systems during experiment, and external B-dot probe arrays to measure radiated magnetic fields on the firing table. Both of these diagnostics assist us in verifying successful operation the FCG and bunker systems during the experiment.

\section{RECENT EXPERIMENTAL RESULTS}

To date, we have fired six FPGs and two Mini-G's at the Bunker 851 facility. Below are selected photos and data from several of these experiments. 

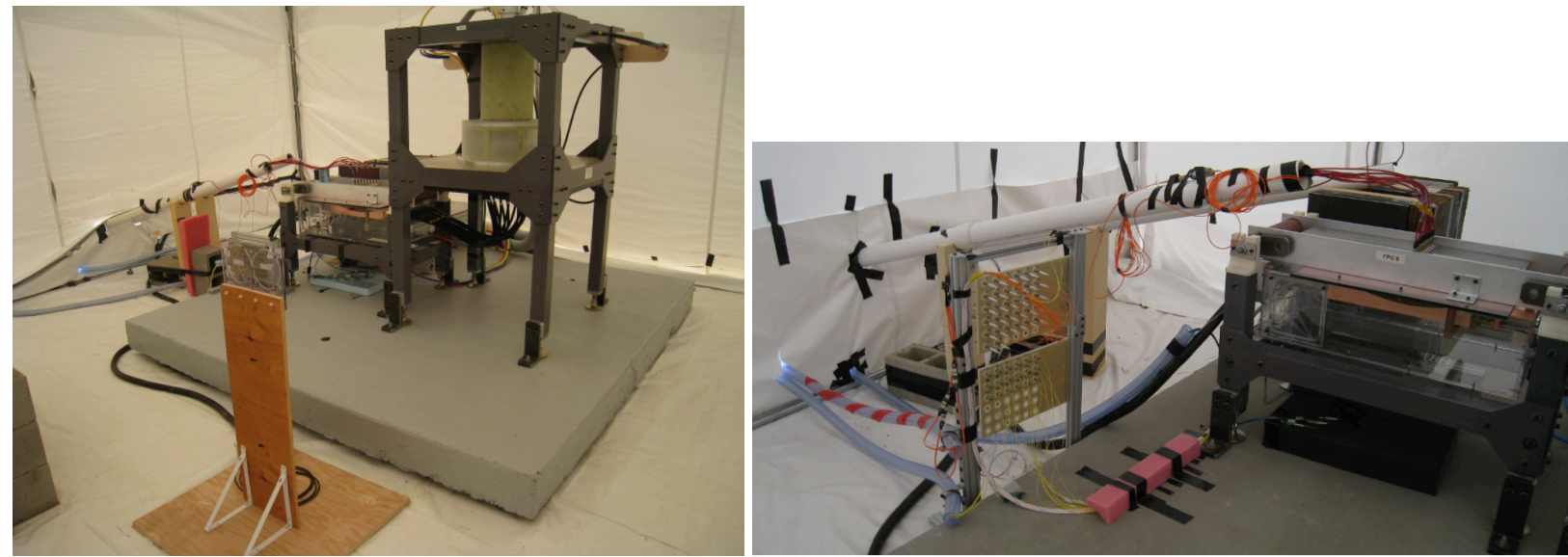

Figure 5. Photos of FPGs assembled on the shot table, minutes before execution. (left) The helical generator is upright and to the right. In the foreground, mounted to plywood, is an external B-dot probe array used to measure leakage flux during the experiment. (right) the flat plate generator is visible, as is an optical patch panel used for diagnostics.
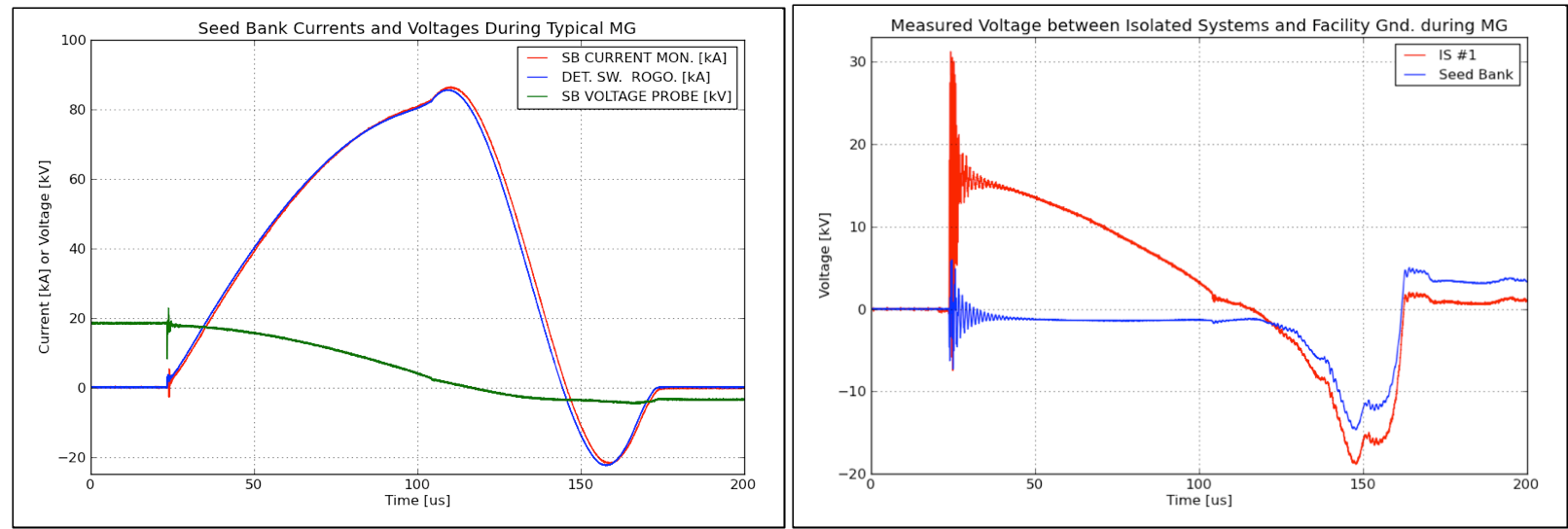

Figure 6. (left) Plots of seed bank current and voltages, and (right) the large voltages developed between the seed bank and facility ground, and IS \#1 and facility ground during a typical Mini-G experiment. In both plots, seed bank discharge begins around 23 us, and helical generator crowbar occurs around 105 us.
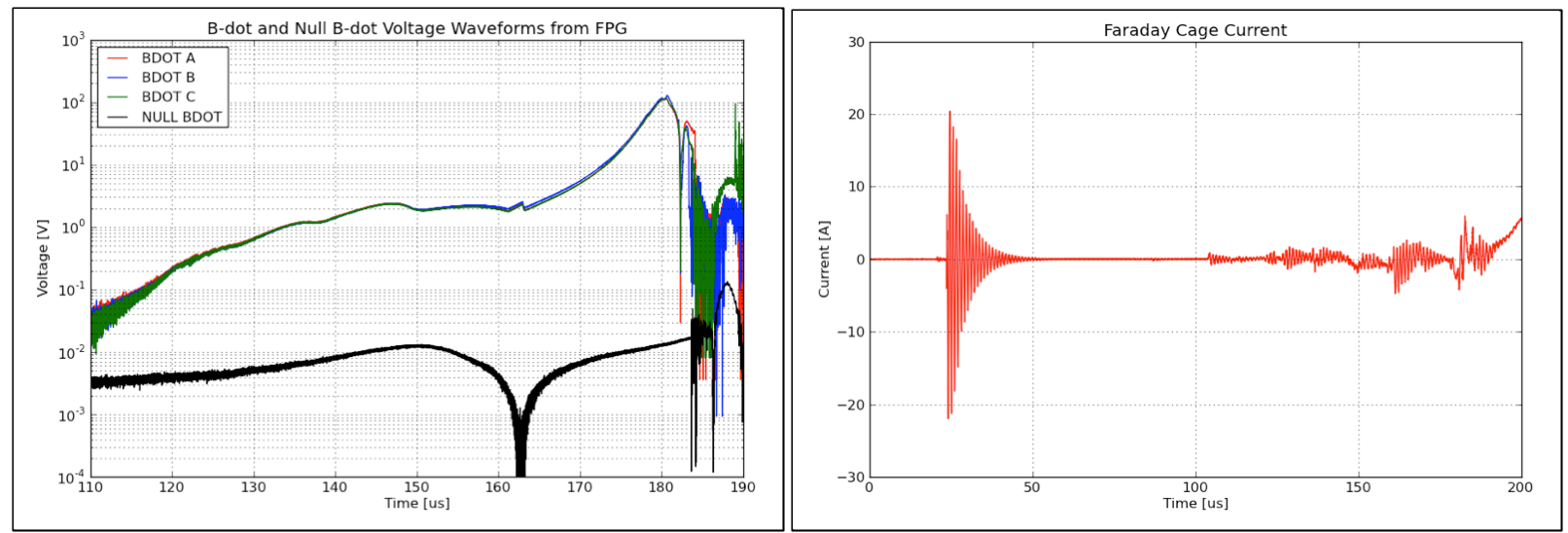

Figure 7. (left) Typical B-dot traces from a FPG, including a null B-dot trace. The plot is shown on logarithmic amplitude scale to emphasive the difference in amplitude between the nominal B-dot signals and the null signal. This difference is roughly two to four orders of magnitude during the time of interest. (right) Measured facility Faraday cage current during a FPG. The initial oscillation occurs during the seed bank discharge. The next oscillation at approximately 105 us occurs when the helical generator crowbars. The current injected into the Faraday cage is low during generator operation-less than 10 amps. 


\section{FACILITY RELIABILITY AND TIMING StATISTICS}

In the past 14 months, we have performed eight HEPP experiments at Bunker 851, and dozens of dry-runs and system tests using selected systems. We have exercised system components sufficiently to derive timing statistics for them, which are shown in Fig. 8.

We have observed no failures of any critical HEPP system component on any dry-run or experiment, and can therefore not yet derive any particularly useful statistics for reliability.

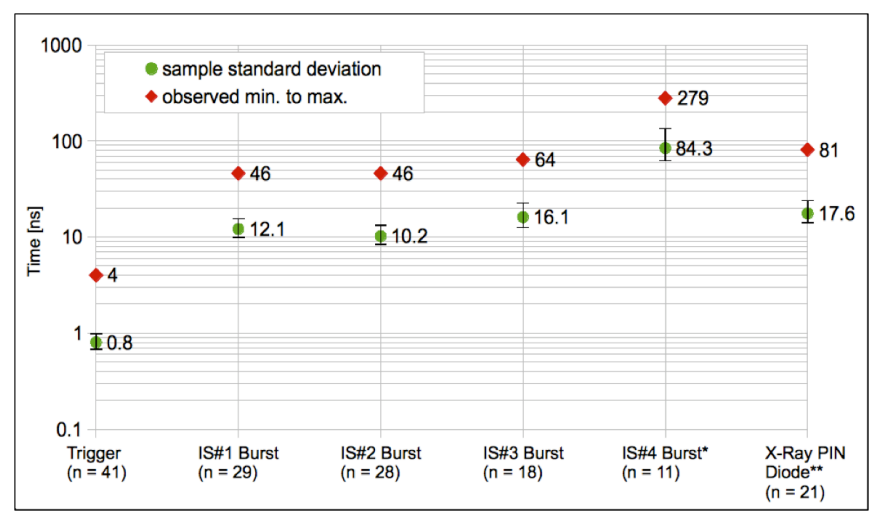

Figure 8. Plot of timing statistics for several key systems. The error bars denote the $95 \%$ confidence interval, derived with a chi-squared test using the number of samples $n$ indicated below each category. *The timing statistics for IS\#4 were gathered across several lots of EBWs, and no effort was made to correct for this. Jitter within any single lot is lower than the cross-lot numbers shown here. **X-ray PIN timing is affected systematically by Marx voltage; the Marx voltage was varied during these observations but no efforts were made to correct for this in the statistics shown here

\section{ACKNOWLEDGMENT}

This work performed under the auspices of the U.S. Department of Energy by Lawrence Livermore National Laboratory under Contract DE-AC52-07NA27344.

\section{REFERENCES}

[1] D. A. Goerz et al., "Flat Plate FCG Experimental System for Material Studies," these proceedings.

[2] D. B. Reisman et al., "Explosive Flux Compression Generators at LLNL," these proceedings.

[3] D.B. Reisman et al., "The advanced helical generator", Rev. Sci. Instr., 81, 2010.

[4] D.B. Reisman et al., "The full-function test", Rev. Sci. Instr., 81, 2010.

[5] A. D. White et al., "Measuring helical FCG voltage with an electric field antenna," proc. $18^{\text {th }}$ International Pulsed Power Conference, 2011, pp. $499-503$

[6] D. A. Goerz et al., "Implementation of pulsed power diagnostics on explosive flux compression generators at LLNL," The 13th International Conference on Megagauss Magnetic Field Generation and Related Topics, Suzhou, China, 2010.

[7] A. D. White, G. B. McHale, and D. A. Goerz, "Advances in optical fiber-based Faraday rotation diagnostics," proc. 17th IEEE Pulsed Power Conference, Washington, DC, 2009, pp. 1358-1363.

[8] Coastal Connections, 2368 Eastman Ave, Unit 11, Ventura, CA 93003

[9] O. T. Strand, D. R. Goosman, C. Martinez, T. L. Whitworth, W. W. Kuhlow, "Compact system for high-speed velocimetry heterodyne techniques," Rev. Sci. Instr., 77, 2005

[10] E. P. Daykin, A. Diaz, C. Gallegos, C. Perez, A Rutkowski, “A multiplexed many-point PDV - techniques and technologies," $5^{\text {th }}$ Annual PDV Workshop, Ohio, 2010

[11] ZTEC Instruments, 7715 Tiburon St, Albuquerque, NM 87109 